\title{
A Generalized Approach for Rapid Aqueous MOF Synthesis by Controlling Solution pH
}

Luke Huelsenbeck, Hongxi Luo, Prince Verma, Jillian Dane, Rachel Ho, Emily Beyer, Hailey

Hall, Geoffrey M. Geise, Gaurav Giri*

Department of Chemical Engineering, University of Virginia, Charlottesville, Virginia

\section{Table of Contents}

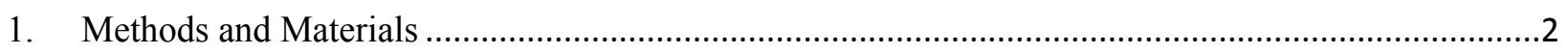

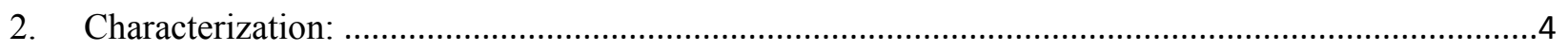

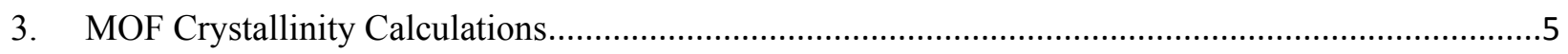

4. Tabulated Conversion, STY, and synthesis method of published rapid syntheses ............................7

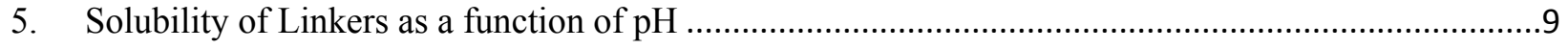

6. Thermogravimetric Analysis of rapid and control syntheses.....................................................10

7. Nitrogen Uptake Isotherms for Rapid Trials ...............................................................................13

8. Scanning Electron Microscopy Images of UiO-66- $\mathrm{NH}_{2}$ Fabric ..................................................14

9. Thermogravimetric Analysis of UiO-66 $\mathrm{NH}_{2}$ Loading on Cotton Fabric........................................15

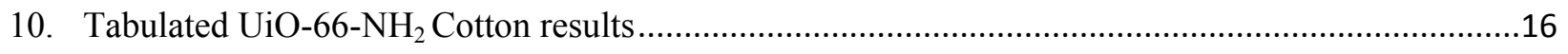

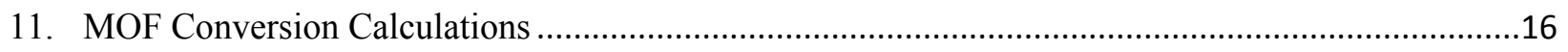

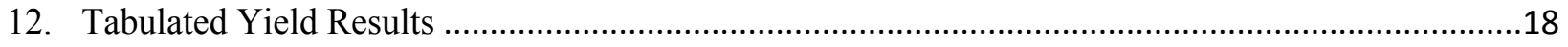

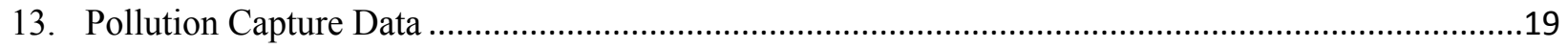

14. Supplementary Information References …..............................................................................19 


\section{Methods and Materials}

Materials:

All reagents were used as received with no further purification. Zirconyl chloride octahydrate $\left(\mathrm{ZrOCl}_{2} \cdot 8\right.$ $\mathrm{H}_{2} \mathrm{O}, 98 \%$ Alfa Aesar), acetic acid (Glacial Sigma Aldrich), sodium hydroxide pellets ( $\mathrm{NaOH}, 98 \%$ Sigma Aldrich), 2-aminoterephthalic acid ( $\mathrm{H}_{2} \mathrm{ATA}, 99 \%$ Sigma Aldrich), terephthalic acid $\left(\mathrm{H}_{2} \mathrm{BDC}\right.$, $98 \%$ Sigma Aldrich) and deionized water were used for the rapid synthesis of UiO-66- $\mathrm{NH}_{2}$ and $\mathrm{UiO}-66$. Copper nitrate hemipentahydrate $\left(\mathrm{Cu}\left(\mathrm{NO}_{3}\right)_{2}, 98 \%\right.$ Sigma Aldrich), hydrochloric acid ( $\mathrm{HCl}, 37 \mathrm{wt} \%$ Alfa Aesar), sodium hydroxide ( $\mathrm{NaOH}, 98 \%$ Sigma Aldrich), and trimesic acid $\left(\mathrm{H}_{3} \mathrm{BTC}\right.$, 95\% Sigma Aldrich), were used for the rapid synthesis of HKUST-1. Zinc nitrate hexahydrate $\left(\mathrm{Zn}\left(\mathrm{NO}_{3}\right)_{2}, 98 \%\right.$ Sigma Aldrich), sodium hydroxide, 2-methylimidazole (HmIm, 97\% Alfa Aesar) were used for the rapid synthesis of ZIF-8. Hydrochloric acid (37 wt \%, Alfa Aesar) was used to prepare an aqueous solution of $0.01 \mathrm{M} \mathrm{HCl}$. Dimethyl sulfoxide (DMSO, 99\% Sigma Aldrich), ethanol (EtOH, 95\% Koptec), and methanol (MeOH, 99.8\% Sigma Aldrich) were used for washing and activating MOFs. Napped Sateen Cotton fabric was purchased from Hanes (Hanes 0339061) and washed with an ethanol rinse before further processing.

\section{Rapid Synthesis of MOFs:}

In a typical synthesis, a metal ion solution and a linker solution were prepared as described below. $10 \mathrm{~mL}$ of linker solution was placed in a $50 \mathrm{~mL}$ conical centrifuge tube and weighed. The $\mathrm{pH}$ was adjusted with a predetermined volume of $20 \mathrm{M} \mathrm{NaOH}$ (between 0 and $3500 \mu \mathrm{L}$ ) and DI water to a total solution volume of $13.5 \mathrm{~mL}$ in the linker solution or by adding a predetermined volume of $37 \mathrm{wt} \% \mathrm{HCl}$ to the metal solution and adjusting the linker solution with DI water to maintain a constant mixture volume. Next, 7.5-10 mL metal solution (weighed previously) was vigorously injected into the linker solution using a $10 \mathrm{~mL}$ pipette. The resulting mixture of metal and linker solutions was immediately placed in a centrifuge at $12000 \mathrm{rpm}$ for 5 minutes. Afterwards, supernatant was carefully decanted to a separate container and $\mathrm{pH}$ was measured. Except for HKUST-1, precipitate was placed in an oven at $70{ }^{\circ} \mathrm{C}$ for a minimum of 8 hours then washed with various solvents depending on the MOF and dried before characterization. Individual MOF treatments are outlined below. After synthesis and washing, all MOFs were stored under nitrogen between characterization.

\section{Rapid Synthesis of UiO-66- $\mathrm{NH}_{2}$ :}

A metal solution was prepared following synthesis outlined by Szilagyi et al. ${ }^{1}$ Briefly, $1.2880 \mathrm{~g} \mathrm{ZrOCl}_{2}$ $\cdot 8 \mathrm{H}_{2} \mathrm{O}$ was dissolved in $5 \mathrm{~mL}$ Acetic acid and $12 \mathrm{~mL}$ of DI water, placed in a Teflon lined $20 \mathrm{~mL}$ scintillation vial, heated at $70{ }^{\circ} \mathrm{C}$ for two hours and allowed to cool to room temperature. The linker solution was prepared by mixing $0.7240 \mathrm{~g}$ of $\mathrm{H}_{2} \mathrm{ATA}$ in a solution of $0.32 \mathrm{~g} \mathrm{NaOH}$ in $20 \mathrm{~mL}$ DI water until dissolved. After centrifugation and drying of the solution containing the precipitated product, the sediment was washed with a $0.01 \mathrm{M} \mathrm{HCl}$ solution, $0.001 \mathrm{M} \mathrm{NaOH}$ solution, DMSO and methanol to remove unreacted and soluble components of the precipitate. A solvent exchange with methanol was performed overnight before additional centrifugation and drying at $70{ }^{\circ} \mathrm{C}$ overnight.

\section{Rapid Synthesis of UiO-66:}

Solutions were prepared and washed similarly to UiO-66- $\mathrm{NH}_{2}$ Briefly, $1.288 \mathrm{~g} \mathrm{ZrOCl} \cdot 8 \mathrm{H}_{2} \mathrm{O}$ was dissolved in $3 \mathrm{~mL}$ Acetic acid and $12 \mathrm{~mL}$ of DI water, placed in a Teflon lined $20 \mathrm{~mL}$ scintillation vial, heated at 70

${ }^{0} \mathrm{C}$ for two hours and allowed to cool to room temperature. The linker solution was prepared by mixing $0.6640 \mathrm{~g}$ of $\mathrm{H}_{2} \mathrm{BDC}$ in a solution of $0.32 \mathrm{~g} \mathrm{NaOH}$ in $20 \mathrm{~mL}$ DI water until dissolved. After centrifugation 
and drying of the solution containing the precipitated product, the sediment was washed and dried using the same procedure as $\mathrm{UiO}-66-\mathrm{NH}_{2}$.

\section{Rapid Synthesis of HKUST-1:}

A metal solution was prepared with $0.4652 \mathrm{~g} \mathrm{Cu}\left(\mathrm{NO}_{3}\right)_{2} \cdot 2.5 \mathrm{H}_{2} \mathrm{O}$ was dissolved in $10 \mathrm{~mL}$ of DI water. The linker solution was prepared by mixing $0.2802 \mathrm{~g}$ of $\mathrm{H}_{3} \mathrm{BTC}$ in a solution of $0.16 \mathrm{~g} \mathrm{NaOH}$ in $10 \mathrm{~mL}$ DI water until dissolved. After centrifugation of the solution containing the precipitated product, the precipitate was immediately washed and solvent exchanged with ethanol to prevent HKUST-1 degradation from water. Samples were dried under vacuum $(30 \mathrm{mmHg})$ at $90^{\circ} \mathrm{C}$ in a nitrogen atmosphere.

\section{Rapid Synthesis of ZIF-L:}

A metal solution was prepared with $0.5950 \mathrm{~g} \mathrm{Zn}\left(\mathrm{NO}_{3}\right)_{2} \cdot 6 \mathrm{H}_{2} \mathrm{O}$ was dissolved in $10 \mathrm{~mL}$ of DI water. The linker solution was prepared by mixing $0.3284 \mathrm{~g}$ of $\mathrm{HmIm}$ in $10 \mathrm{~mL}$ DI water until dissolved. After centrifugation and drying of the solution containing the precipitated product, the sediment was stored in nitrogen atmosphere until characterization.

\section{Synthesis of Cotton@UiO-66- $\mathrm{NH}_{2}$ Fabric:}

Fabric was cut into $5 \mathrm{~cm}$ diameter circles and soaked in ethanol and dried at $80{ }^{\circ} \mathrm{C}$ to clean. MOF was applied by using sequential dip coating (SQD). Dried cloth was dipped in a UiO-66- $\mathrm{NH}_{2}$ metal solution for $\sim 5$ seconds, gently dried with a paper towel to remove excess solution, then dipped in a linker solution 5 seconds. The linker solution used corresponded to optimum conditions found in the $\mathrm{pH}$ study $(0.3620$ $\mathrm{H}_{2} \mathrm{ATA}, 13.5 \mathrm{~mL}$ DI water and $0.4904 \mathrm{~g} \mathrm{NaOH}$ ). After a metal and linker dip, fabric was submerged in DI water and sonicated to remove any unattached particles. The dipping process was repeated up to 12 times to vary the amount of MOF loaded onto fabric. Fabrics were dried at $120{ }^{\circ} \mathrm{C}$ overnight in a convection oven and stored for characterization.

\section{Flash Freezing Study of MOF reaction extent as a function of time:}

For short time points (1-10 seconds), a microfluidic device was used to combine metal and linker solutions at and mixed using a sonicator. Residence time in the microfluidic device (Fittings,Masterflex, FEP tubing, Altaflow) was taken as the reaction time and effluent was the reaction stream was immediately captured in liquid nitrogen to freeze the suspension. Samples were stored at $-80{ }^{\circ} \mathrm{C}$ until lyophilization. No washing was performed before PXRD. After data collection, samples were washed with a $0.01 \mathrm{M} \mathrm{HCl}$ solution, DI water, DMSO and methanol to remove unreacted and soluble components of the precipitate and additional PXRD data was collected of washed samples.

\section{Linker Solubility Study:}

Linker solutions of $0.2 \mathrm{M} \mathrm{H}_{2} \mathrm{ATA}$ and $\mathrm{H}_{2} \mathrm{BDC}$ were prepared by mixing equinormal sodium hydroxide and linker with DI water. Solution $\mathrm{pH}$ was adjusted using $10 \mathrm{M} \mathrm{NaOH}$ and $6 \mathrm{M} \mathrm{HCl}$ under vigorous stirring. Precipitation of the linker was observed with each addition of $\mathrm{NaOH}$ solution, indicating saturation. Solution $\mathrm{pH}$ was allowed to equilibrate before a $5 \mathrm{~mL}$ aliquot was vacuum filtered and vacuum dried at 80 ${ }^{0} \mathrm{C}$ for 4 hours. Dried precipitate was weighed and converted to molarity using the molar mass of the respective organic acid. 


\section{Characterization:}

pH Measurement: $\mathrm{pH}$ Measurements were recorded using a Mettler Toledo SevenCompact pH meter S220 and an InLab Expert Pro ISM electrode ( $\mathrm{pH}$ range 0-14). The probe was calibrated using a multipoint calibration with buffer solutions of $\mathrm{pH} 2.00,4.01,7.00,9.21,11.00$ (Mettler Toledo). The probe was thoroughly rinsed between readings with DI water and dried before subsequent readings.

Weight and Conversion: Individual linker solution weight and averaged metal solution weight were used to calculate the quantity of reactants in each synthesis. Since MOF stoichiometric values of reactants were used, the total weight of metal and linker were used in calculating percent conversion. Centrifuge tubes used for synthesis were weighed before use and precipitate weight was determined as weight of empty tube plus the dried precipitate.

XRD: Powder x-ray diffraction (PXRD) patterns were collected using a PANalytical Empyrean X-ray Diffractometer (Malvern Panalytical, Egham, UK) with $\mathrm{Cu}$ K- $\alpha$ radiation. Scans were taken from $2 \theta=5^{\circ}$ $20^{\circ}$ for UiO-66- $\mathrm{NH}_{2}$ and $2 \theta=5^{\circ}-30^{\circ}$ for UiO-66, HKUST-1, and ZIF-L. Diffraction patterns were analyzed using HighScore Plus X-ray Diffraction analysis software.

SEM: Scanning Electron Microscopy (SEM) micrographs were collected using a Helios Dual Beam FIB G4 UC with an accelerating voltage of $5 \mathrm{kV}$ and $0.80 \mathrm{nA}$ current.

BET: Nitrogen uptake isotherms were measured at $77 \mathrm{~K}$ with a Micromeritics ASAP 2020 Surface Area and Porisimetry analyzer. Data was analyzed using the ASAP 2020 V4.04 software. All gases used were high purity. Typically, 50-100 mg of MOF sample were dried under vacuum at $80^{\circ} \mathrm{C}$ overnight before initiating previously reported degassing procedures for each MOF. ${ }^{2-5}$

TGA: The metal to linker ratio of MOFs was measured by the Thermogravimetric Analyzer (TGA, Q50). In a typical experiment, a small amount of predried powder or fabric $(\sim 10 \mathrm{mg})$ was loaded into a Pt sample pan, and the sample pan was transferred into the heating chamber of the analyzer. The sample gas was air (flowrate $=60 \mathrm{~mL} / \mathrm{min}$ ), and the balance gas was nitrogen (flowrate $=40 \mathrm{~mL} / \mathrm{min}$ ). The sample was heated to $800{ }^{\circ} \mathrm{C}$ from room temperature at a rate of $10^{\circ} \mathrm{C} / \mathrm{min}$. In the initial stage of the heating process, moisture adsorbed by the sample was evaporated, and the sample weight at $120^{\circ} \mathrm{C}$ was taken as the dry weight. Further heating led to the decomposition of the sample (i.e., organic linkers and organic fabric were decomposed by oxidation and metal nodes were transformed into metal oxide), and the sample weight at $800{ }^{\circ} \mathrm{C}$ was taken as the weight of metal oxide.

Pollution capture: A multichamber pollution capture device was used to measure capture efficiency of particles from PM 1-4. Incense (1/8" diameter, $1 / 2$ " long, local market was ignited and smoke was blown into a holding chamber. Smoke was dosed and dispersed from the holding chamber into a controlled chamber to maintain a particle concentration of $\sim 500 \mu \mathrm{g} / \mathrm{m}^{3}$ using electric fans, vacuum pumps and a microcontroller-based pollution sensor (Microcontroller: Arduino Uno, Arduino, Pollution Sensor: PMS5003, Adafruit). Smoke was then pulled through a filter (cross section: $3.14 \mathrm{~cm}^{2}$ ) or directly from the chamber at 1 LPM into an Aerosol monitor (pDR-1500 with PM 1-4 cutpoint, ThermoFisher Scientific) to record the particulate matter concentration. Pressure drop was measured with a differential pressure gauge (Magnehelic) tapped into the upstream and downstream sides of the filter. The capture efficiency was calculated as the average concentration over 60 minutes with the test filter over the average concentration over 60 minutes with no filter. 


\section{MOF Crystallinity Calculations}

$\%$ Crystallinity is determined by comparing the crystalline scattering intensities to all scattering from the sample using the HighScore Plus X-ray Diffraction analysis software. ${ }^{6}$ In this analysis we assume the background and incoherent scattering are negligible within the scattering angles used. It should be noted this assumption provides a lower bound of crystallinity by increasing the denominator. Additionally, we assume the chemical composition and density are approximately the same for precipitates showing a measurable degree of MOF crystallinity, as these are likely amorphous coordination polymers. This is evidenced by the absence of non-MOF diffraction peaks and similar solubility characteristics of the precipitate to MOF. Given crystalline MOF product is our desired species, we use crystallinity as a proxy to obtain the reaction selectivity. The crystallinity is calculated by integrating over diffraction angles associated with several MOF planes and the total signal as follows:

$$
\% \text { Crystallinity }=\frac{\int_{2 \theta_{1}}^{2 \theta_{2}} I_{c r} d(2 \theta)}{\int_{2 \theta_{1}}^{2 \theta_{2}}\left(I_{c r}+I_{a m}\right) d(2 \theta)}
$$

The following demonstrates how $I_{c r}$ and $I_{a m}$ were determined for a UiO-66 $\mathrm{NH}_{2}$ sample using a background subtraction in HighScore Plus:

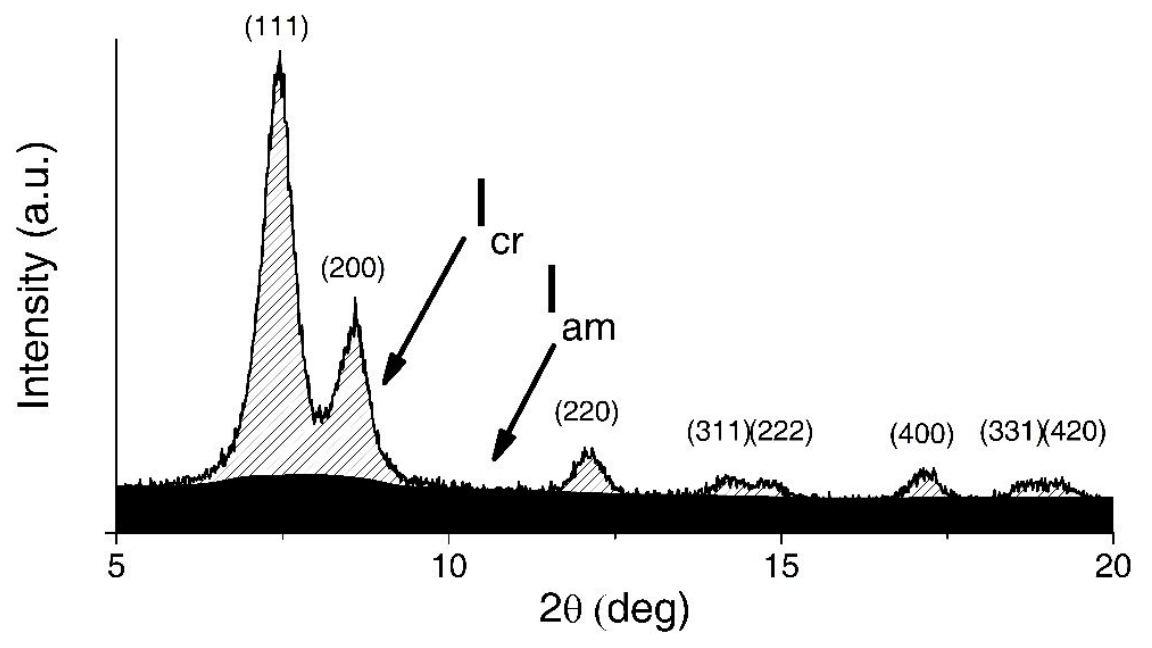

Figure S1. Schematic showing \% crystallinity analysis comparing the area under diffraction curve due to crystalline diffraction $\left(I_{c r+}\right)$ vs. amorphous $\left(I_{a m}\right)$ scattering. Amorphous region is shown as black and crystalline region is shown as the hashed region. 

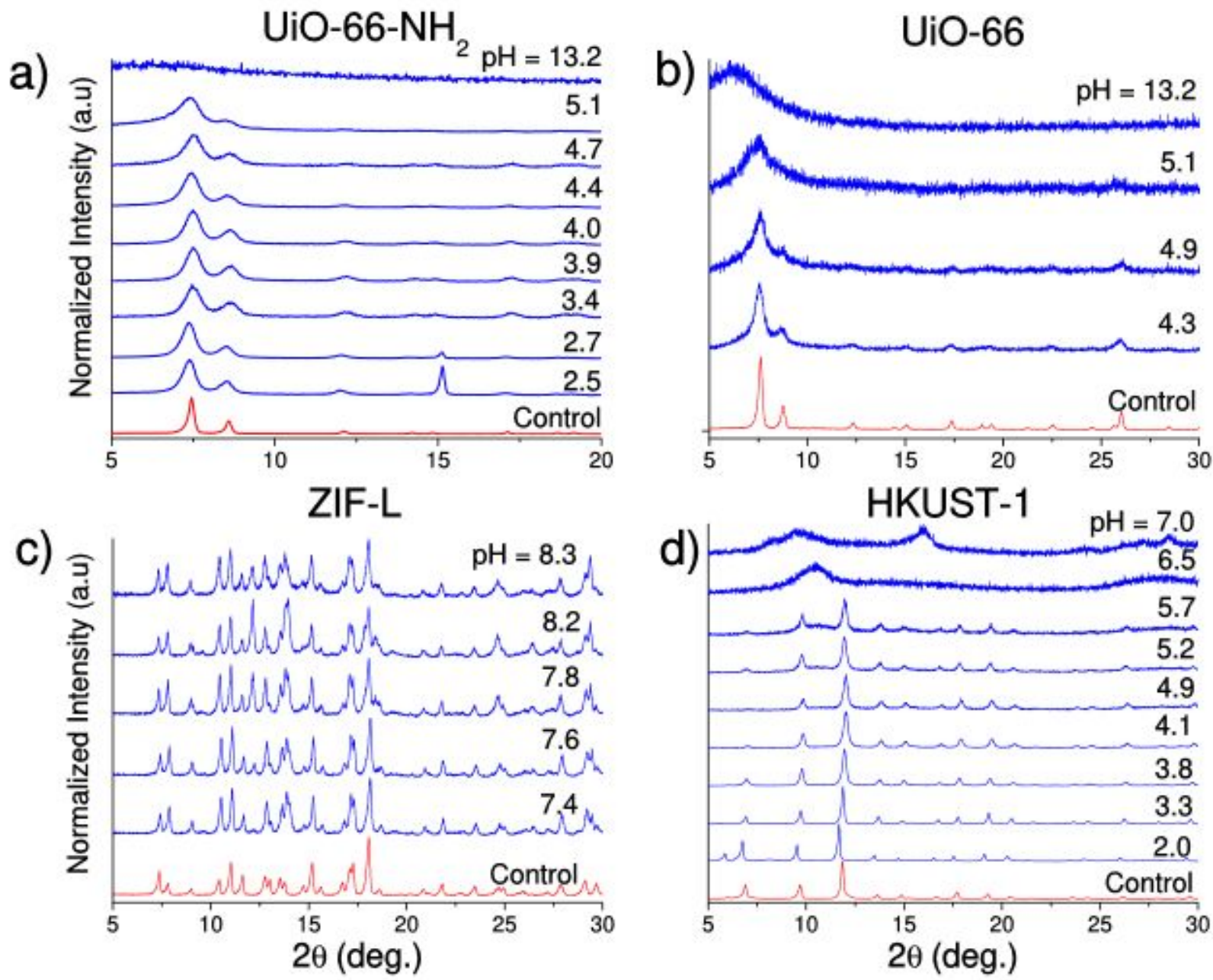

Figure S2. Typical PXRD diffraction patterns used to determined \% crystallinity as a function of pH for a) UiO-66-NH $\mathrm{H}_{2}$ b) UiO66 c) ZIF-L and d) HKUST-1. Crystallinity is assumed to be $100 \%$ if conversion provided insufficient material for PXRD after washing, representing an upper bound for MOF obtained. 


\section{Tabulated Conversion, STY, and synthesis method of published rapid syntheses}

Space time yield calculation for solvothermal synthesis:

$$
S T Y=\frac{\text { Washed and Dried Precipitate Mass }(\mathrm{kg})}{\text { Synthesis Time }(\text { days }) * \text { Synthesis Volume }\left(\mathrm{m}^{3}\right)}
$$

Table S1. Table of conversion, space time yield (STY), synthesis method and conditions for rapid production of prototypical MOFs

\begin{tabular}{|c|c|c|c|c|c|}
\hline MOF & Conversion & $\begin{array}{l}\text { STY }(\mathrm{kg} \\
\left.\mathrm{m}^{-3} \mathrm{day}^{-1}\right)\end{array}$ & Method & Conditions & Source \\
\hline $\mathrm{UiO}-66-\mathrm{NH}_{2}$ & $96 \%$ & 3381 & This work & $10 \min , \mathrm{RT}, \mathrm{H}_{2} \mathrm{O}$ & $\begin{array}{l}\text { This } \\
\text { work }\end{array}$ \\
\hline $\mathrm{UiO}-66-\mathrm{NH}_{2}$ & ca. $70 \%$ & 2679 & Microwave & Continuous, $85{ }^{\circ} \mathrm{C}, \mathrm{H}_{2} \mathrm{O}$ & 7 \\
\hline $\mathrm{UiO}-66-\mathrm{NH}_{2}$ & ca. $100 \%$ & 716 & Aqueous & $1 \mathrm{hr}, \mathrm{RT}, \mathrm{H}_{2} \mathrm{O}$ & 1 \\
\hline $\mathrm{UiO}-66-\mathrm{NH}_{2}$ & $105 \%$ & 43 & Solvothermal & $18 \mathrm{hr}, 120^{\circ} \mathrm{C}, \mathrm{DMF}$ & 8 \\
\hline $\mathrm{UiO}-66-\mathrm{NH}_{2}$ & $122 \%$ & 37 & Solvothermal & $24 \mathrm{hr}, 120^{\circ} \mathrm{C}, \mathrm{DMF}$ & 8 \\
\hline $\mathrm{UiO}-66-\mathrm{NH}_{2}$ & $97 \%$ & 21 & Solvothermal & $24 \mathrm{hr}, 120{ }^{\circ} \mathrm{C}, \mathrm{DMF}$ & 8 \\
\hline $\mathrm{UiO}-66-\mathrm{NH}_{2}$ & $68 \%$ & 21 & Solvothermal & $24 \mathrm{hr}, 120^{\circ} \mathrm{C}, \mathrm{DMF}$ & 8 \\
\hline $\mathrm{UiO}-66-\mathrm{NH}_{2}$ & $105 \%$ & 16 & Solvothermal & $24 \mathrm{hr}, 120{ }^{\circ} \mathrm{C}, \mathrm{DMF}$ & 8 \\
\hline $\mathrm{UiO}-66-\mathrm{NH}_{2}$ & $39 \%$ & 12 & Solvothermal & $24 \mathrm{hr}, 120{ }^{\circ} \mathrm{C}, \mathrm{DMF}$ & 8 \\
\hline $\mathrm{UiO}-66-\mathrm{NH}_{2}$ & $70 \%$ & 10 & Spray Drying & Continuous, $90{ }^{\circ} \mathrm{C}, \mathrm{H}_{2} \mathrm{O}$ & 9 \\
\hline UiO-66 & $79 \%$ & 2732 & This Work & $10 \mathrm{~min}, \mathrm{RT}, \mathrm{H}_{2} \mathrm{O}$ & $\begin{array}{l}\text { This } \\
\text { work }\end{array}$ \\
\hline $\mathrm{UiO}-66$ & $67 \%$ & 672 & Flow Reactor & $\begin{array}{c}\text { Continuous, } 130{ }^{\circ} \mathrm{C} \text {, } \\
\text { DMF }\end{array}$ & 10 \\
\hline $\mathrm{UiO}-66$ & $90 \%$ & 53 & Solvothermal & $24 \mathrm{hr}, 120^{\circ} \mathrm{C}, \mathrm{DMF}$ & 8 \\
\hline UiO-66 & $90 \%$ & 43 & Microwave & $2 \mathrm{hr}, 100{ }^{\circ} \mathrm{C}, \mathrm{DMF}$ & 11 \\
\hline UiO-66 & $115 \%$ & 33 & Solvothermal & $24 \mathrm{hr}, 120^{\circ} \mathrm{C}, \mathrm{DMF}$ & 8 \\
\hline $\mathrm{UiO}-66$ & $151 \%$ & 30 & Solvothermal & $12 \mathrm{hr}, 80{ }^{\circ} \mathrm{C}, \mathrm{DMF}$ & 3 \\
\hline $\mathrm{UiO}-66$ & $90 \%$ & 26 & Solvothermal & $24 \mathrm{hr}, 120^{\circ} \mathrm{C}, \mathrm{DMF}$ & 8 \\
\hline UiO-66 & $55 \%$ & 21 & Solvothermal & $18 \mathrm{hr}, 120{ }^{\circ} \mathrm{C}, \mathrm{DMF}$ & 8 \\
\hline UiO-66 & $70 \%$ & 12 & Spray Drying & $\begin{array}{c}\text { Continuous, } 115{ }^{\circ} \mathrm{C} \text {, } \\
\text { DMF }+\mathrm{H}_{2} \mathrm{O}\end{array}$ & 9 \\
\hline $\mathrm{UiO}-66$ & $60 \%$ & 4 & Spray Drying & $\begin{array}{c}\text { Continuous, } 180^{\circ} \mathrm{C}, \\
\text { DMF }\end{array}$ & 12 \\
\hline HKUST-1 & $86 \%$ & 2288 & This Work & $10 \mathrm{~min}, \mathrm{RT}, \mathrm{H}_{2} \mathrm{O}$ & $\begin{array}{l}\text { This } \\
\text { work }\end{array}$ \\
\hline HKUST-1 & $98 \%$ & 36000 & Hydroxy Double Salt & $1 \mathrm{~min}, \mathrm{RT}, \mathrm{DMF}+\mathrm{H}_{2} \mathrm{O}$ & 13 \\
\hline HKUST-1 & $100 \%$ & 4533 & Plug Flow Reactor & $\begin{array}{c}\text { Continuous, } 140^{\circ} \mathrm{C} \\
\text { EtOH }\end{array}$ & 10 \\
\hline
\end{tabular}




\begin{tabular}{|c|c|c|c|c|c|}
\hline HKUST-1 & N/A & 4399 & $\begin{array}{c}\text { Counter Current } \\
\text { Reactor } \\
\end{array}$ & $\begin{array}{l}\text { Continuous, } 300^{\circ} \mathrm{C}, \\
\mathrm{DMF}+\mathrm{H}_{2} \mathrm{O}+\mathrm{EtOH}\end{array}$ & 14 \\
\hline HKUST-1 & $90 \%$ & 2035 & Aqueous & $1 \mathrm{hr}, \mathrm{RT}, \mathrm{H}_{2} \mathrm{O}$ & 15 \\
\hline HKUST-1 & $64 \%$ & 494 & Aerosol Synthesis & $\begin{array}{c}\text { Continuous, } 160^{\circ} \mathrm{C}, \mathrm{H}_{2} \mathrm{O} \\
+\mathrm{EtOH}\end{array}$ & 16 \\
\hline HKUST-1 & N/A & 225 & Electrochemical & $2.5 \mathrm{hr}, \mathrm{RT}, \mathrm{MeOH}$ & 17 \\
\hline HKUST-1 & $85 \%$ & 30 & Spray Drying & $\begin{array}{l}\text { Continuous, } 120^{\circ} \mathrm{C}, \\
\mathrm{DMF}+\mathrm{H}_{2} \mathrm{O}+\mathrm{EtOH}\end{array}$ & 12 \\
\hline HKUST-1 & $68 \%$ & 5.8 & Microfluidic Droplet & $\begin{array}{c}\text { Continuous, } 90^{\circ} \mathrm{C}, \mathrm{DMF} \\
+\mathrm{H}_{2} \mathrm{O}+\mathrm{EtOH}\end{array}$ & 18 \\
\hline ZIF-L & $103 \%$ & 2879 & This Work & $10 \mathrm{~min}, \mathrm{RT}, \mathrm{H}_{2} \mathrm{O}$ & $\begin{array}{r}\text { This } \\
\text { work }\end{array}$ \\
\hline ZIF-8 & N/A & 3875 & $\begin{array}{c}\text { Counter Current } \\
\text { Reactor }\end{array}$ & $\begin{array}{l}\text { Continuous, } 300^{\circ} \mathrm{C}, \\
\mathrm{DMF}+\mathrm{H}_{2} \mathrm{O}+\mathrm{EtOH}\end{array}$ & 5 \\
\hline ZIF-8 & $80 \%$ & 2337 & Aqueous & $5 \mathrm{~min}, \mathrm{RT}, \mathrm{H}_{2} \mathrm{O}$ & 19 \\
\hline ZIF-8 & N/A & 100 & Electrochemical & $2.5 \mathrm{hr}, \mathrm{RT}, \mathrm{MeOH}$ & 17 \\
\hline ZIF-8 & $17 \%$ & 69.4 & Aerosol Synthesis & $\begin{array}{c}\text { Continuous, } 50^{\circ} \mathrm{C}, \\
\mathrm{MeOH}\end{array}$ & 16 \\
\hline ZIF-8 & $50 \%$ & 67 & Solvothermal & $1 \mathrm{hr}$, RT, Methanol & 13 \\
\hline ZIF-L & $90 \%$ & 39 & Aqueous & $4 \mathrm{hr}, \mathrm{RT}, \mathrm{H}_{2} \mathrm{O}$ & 5 \\
\hline ZIF-L & $90 \%$ & 31 & Aqueous & $1 \mathrm{hr}, \mathrm{RT}, \mathrm{H}_{2} \mathrm{O}$ & 5 \\
\hline ZIF-8 & $10 \%$ & 4 & Spray Drying & Continuous, $180^{\circ} \mathrm{C}, \mathrm{H}_{2} \mathrm{O}$ & 12 \\
\hline
\end{tabular}




\section{Solubility of Linkers as a function of $\mathrm{pH}$}

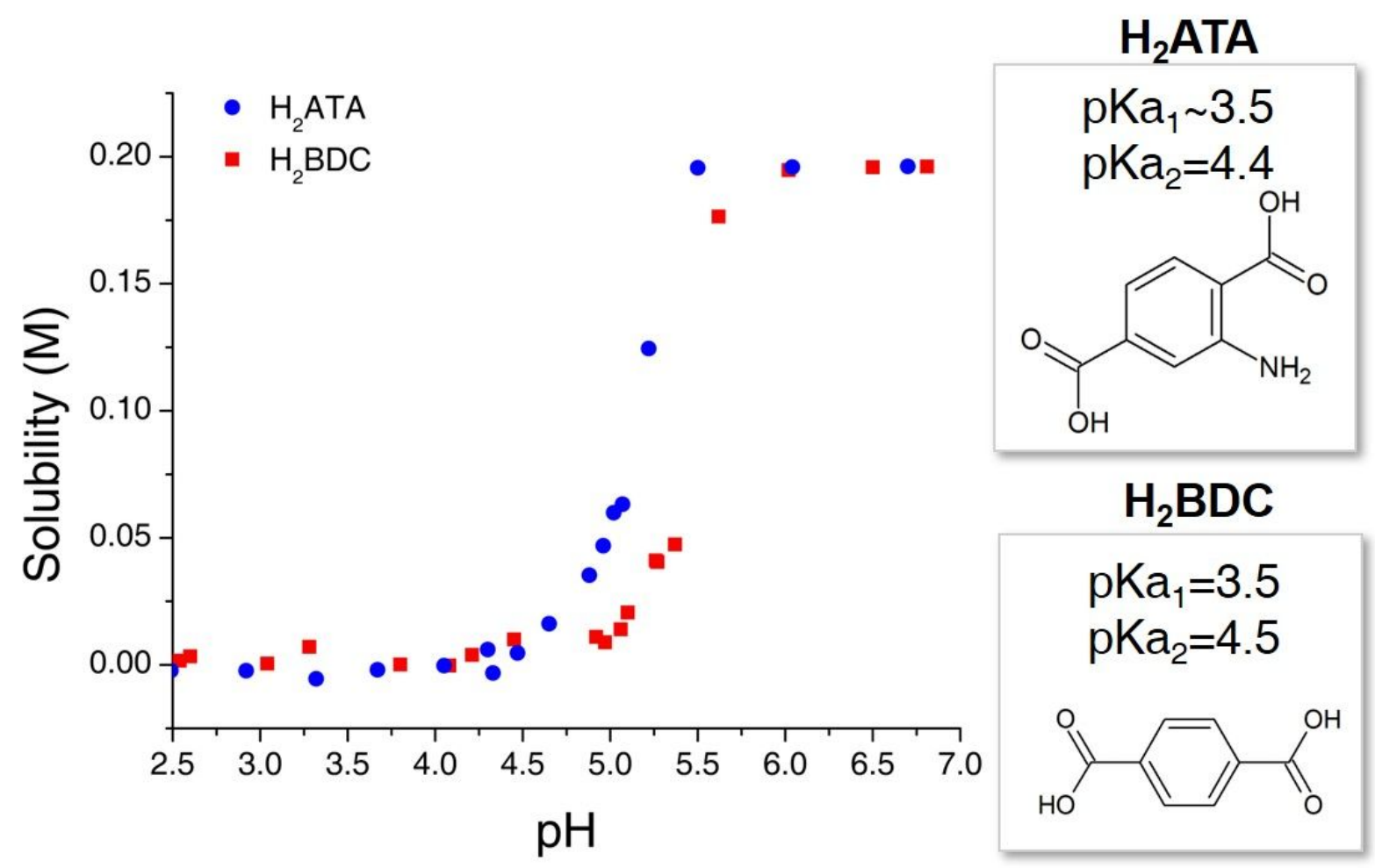

Figure S3. Solubility of $\mathrm{H}_{2} \mathrm{BDC}$ (red) and $\mathrm{H}_{2} \mathrm{ATA}$ (blue) as a function of pH up to $0.2 \mathrm{M}$. It can be seen $\mathrm{H}_{2}$ ATA remains more soluble at lower $\mathrm{pH}$ values indicating a higher concentration of linkers is available for the coordination reaction. 


\section{Thermogravimetric Analysis of rapid and control syntheses}
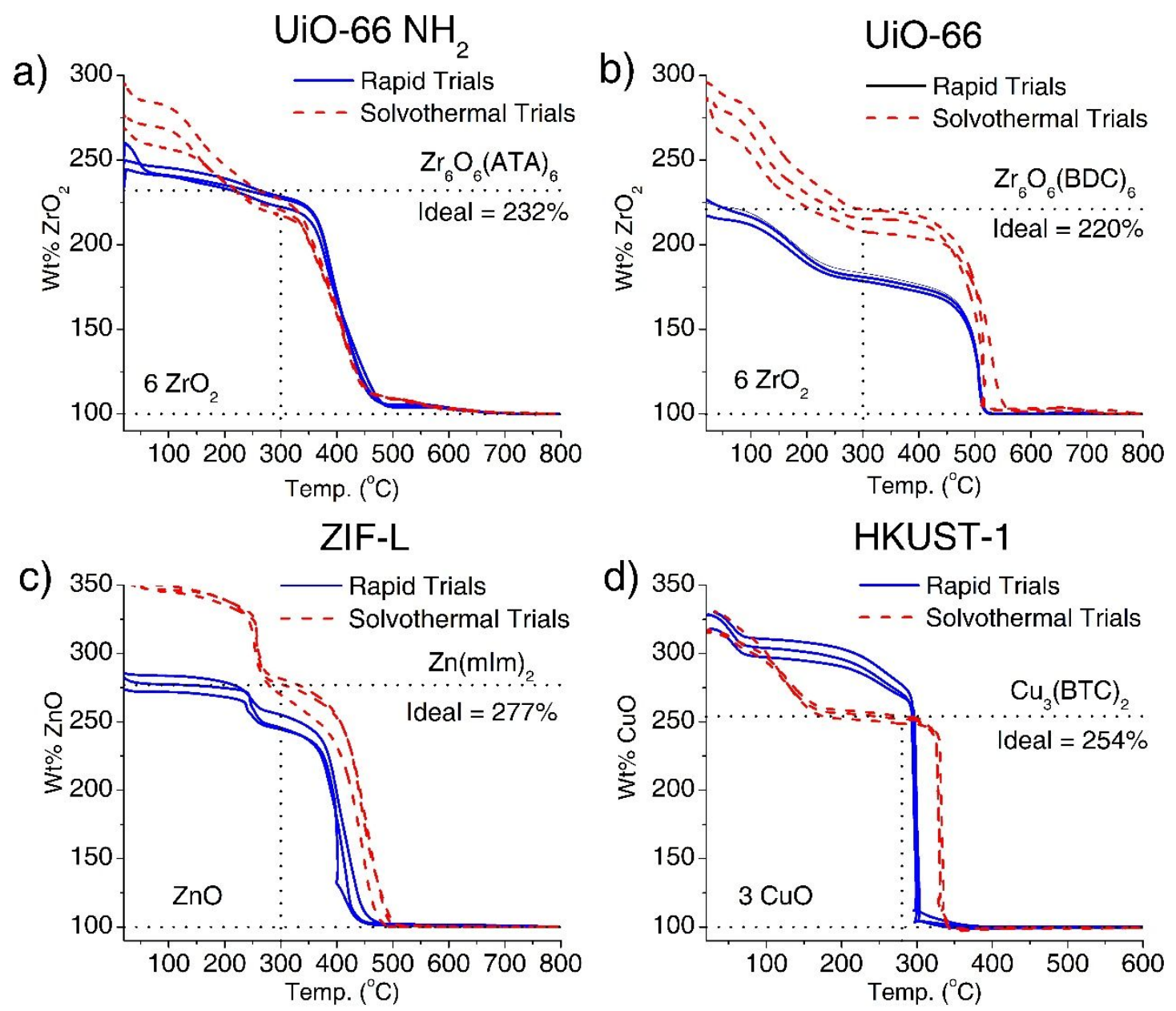

Figure S4. Thermogravimetric decomposition curves of rapidly synthesized (blue) and solvothermal control (dashed red) MOFs of a) UiO-66- $\mathrm{NH}_{2}$ b) UiO-66 c) ZIF-L and d) HKUST-1. N=3. All solvothermal controls exhibited solvent loss up to $300{ }^{\circ} \mathrm{C}$ as shown by the gradual loss of weight. ZIF-L shows a sharp drop in weight around $250^{\circ} \mathrm{C}$ which is the loss of organic HmIm in the structure. The horizontal dashed lines represent the wt\% for ideal stoichiometry (i.e. no missing linker or metal defects). The vertical dashed lines represent the temperature at which the solvent free wt\% is used to calculate metal to organic composition. 
Table S2. Table of equations used to calculate missing linker defects using thermogravimetric analysis results

\begin{tabular}{|c|c|}
\hline $\begin{array}{l}\text { Missing linker defect calculation for } \mathrm{UiO}-66-\mathrm{NH}_{2}: \\
\qquad 226 \%=\frac{\mathrm{Zr}_{6} \mathrm{O}_{6}(\mathrm{ATA})_{x}}{6 \mathrm{ZrO}_{2}}=\frac{(642+179 x) \frac{\mathrm{g}}{\mathrm{mol}}}{739 \frac{\mathrm{g}}{\mathrm{mol}}}\end{array}$ & Eq. 3 \\
\hline $\begin{array}{l}\text { Solving for } 2 \mathrm{x} \text { gives the number of linkers per oxocluster: } \\
\qquad 2 x=2 \cdot \frac{226 \% \cdot 739 \frac{\mathrm{g}}{\mathrm{mol}} 6 \mathrm{ZrO}_{2}-642 \frac{\mathrm{g}}{\mathrm{mol}} \mathrm{Zr}_{6} \mathrm{O}_{6}}{179 \frac{\mathrm{g}}{\mathrm{mol}} \mathrm{ATA}}=11.5 \frac{\mathrm{ATA}}{\mathrm{Zr}_{6} \mathrm{O}_{6}}\end{array}$ & Eq. 4 \\
\hline $\begin{array}{l}\text { Missing linker defect calculation for UiO-66: } \\
\qquad 181 \%=\frac{\mathrm{Zr}_{6} \mathrm{O}_{6}(B D C)_{x}}{6 \mathrm{ZrO}_{2}}=\frac{(642+164 x) \frac{\mathrm{g}}{\mathrm{mol}}}{739 \frac{\mathrm{g}}{\mathrm{mol}}}\end{array}$ & Eq. 5 \\
\hline $\begin{array}{l}\text { Solving for } 2 \mathrm{x} \text { gives the number of linkers per oxocluster: } \\
\qquad 2 x=2 \cdot \frac{181 \% \cdot 739 \frac{\mathrm{g}}{\mathrm{mol}}-642 \frac{\mathrm{g}}{\mathrm{mol}}}{164 \frac{\mathrm{g}}{\mathrm{mol}}}=8.5 \frac{\mathrm{BDC}}{\mathrm{Zr}_{6} \mathrm{O}_{6}}\end{array}$ & Eq. 6 \\
\hline $\begin{array}{l}\text { Missing linker defect calculation for ZIF-L: } \\
\qquad 248 \%=\frac{Z n(m I m)_{x}}{Z n O}=\frac{(65+81 x) \frac{g}{\mathrm{~mol}}}{81 \frac{\mathrm{g}}{\mathrm{mol}}}\end{array}$ & Eq. 7 \\
\hline $\begin{array}{l}\text { Solving for } x \text { gives the number of linkers per zinc node: } \\
\qquad x=\frac{248 \% \cdot 81 \frac{\mathrm{g}}{\mathrm{mol}}-65 \frac{\mathrm{g}}{\mathrm{mol}}}{81 \frac{\mathrm{g}}{\mathrm{mol}}}=1.7 \frac{\mathrm{mIm}}{\mathrm{Zn}}\end{array}$ & $E q .8$ \\
\hline $\begin{array}{l}\text { Added linker defect calculation for HKUST-1: } \\
\qquad 248 \%=\frac{C u_{3}(B T C)_{x}}{3 C u O}=\frac{(192+207 x) \frac{\mathrm{g}}{\mathrm{mol}}}{239 \frac{\mathrm{g}}{\mathrm{mol}}}\end{array}$ & Eq. 9 \\
\hline Solving for $\mathrm{x}$ gives the number of linkers per copper dimer node: & Eq. 10 \\
\hline
\end{tabular}




$$
2 x=\frac{272 \% \cdot 239 \frac{\mathrm{g}}{\mathrm{mol}}-192 \frac{\mathrm{g}}{\mathrm{mol}}}{207 \frac{\mathrm{g}}{\mathrm{mol}}}=4.4 \frac{\mathrm{BTC}}{2 \mathrm{Cu}}
$$

Table S3. Table of thermal decomposition equations used to calculate the ideal weight percent (i.e. defect free) for each MOF and calculations for ideal stoichiometric starting weight percent for thermogravimetric decomposition.

\begin{tabular}{|c|c|}
\hline $\begin{aligned} \mathrm{Zr}_{6} \mathrm{O}_{6}(\mathrm{ATA})_{6}(\mathrm{~s})+(45+3 x) \mathrm{O}_{2}(g) & \rightarrow 6 \mathrm{ZrO}_{2}(s)+48 \mathrm{CO}_{2}(g)+6 \mathrm{NO}_{x}(g)+12 \mathrm{H}_{2} \mathrm{O}(\mathrm{g})\end{aligned}$ & Eq. 11 \\
\hline Ideal Weight Percent $=\frac{\mathrm{Zr}_{6} \mathrm{O}_{6}(A T A)_{6}}{6 \mathrm{ZrO}_{2}}=\frac{1718 \frac{\mathrm{g}}{\mathrm{mol}}}{739 \frac{\mathrm{g}}{\mathrm{mol}}} \times 100 \%=232 \%$ & Eq. 12 \\
\hline $\mathrm{Zr}_{6} \mathrm{O}_{6}(B D C)_{6}(s)+45 \mathrm{O}_{2}(g) \rightarrow 6 \mathrm{ZrO}_{2}(s)+48 \mathrm{CO}_{2}(g)+12 \mathrm{H}_{2} \mathrm{O}(\mathrm{g})$ & Eq. 13 \\
\hline Ideal Weight Percent $=\frac{\mathrm{Zr}_{6} \mathrm{O}_{6}(\mathrm{BDC})_{6}}{6 \mathrm{ZrO}_{2}}=\frac{1628 \frac{\mathrm{g}}{\mathrm{mol}}}{739 \frac{\mathrm{g}}{\mathrm{mol}}} \times 100 \%=220 \%$ & Eq. 14 \\
\hline $\begin{aligned} \mathrm{Zn}(\mathrm{mIm})_{2} \cdot(\mathrm{HmIm})_{\frac{1}{2}} \cdot\left(\mathrm{H}_{2} \mathrm{O}\right) & \frac{3}{2}(\mathrm{~s})+(17.75+3 x) \mathrm{O}_{2}(\mathrm{~g}) \\
& \stackrel{@ 250^{\circ} \mathrm{C}}{\longrightarrow} \mathrm{Zn}(\mathrm{mIm})_{2}(\mathrm{~s})+2 \mathrm{CO}_{2}(\mathrm{~g})+6 \mathrm{NO}_{x}(\mathrm{~g})+7.5 \mathrm{H}_{2} \mathrm{O}(\mathrm{g}) \\
& \stackrel{@ @ 400^{\circ} \mathrm{C}}{\longrightarrow} \mathrm{ZnO}(\mathrm{s})+10 \mathrm{CO}_{2}(\mathrm{~g})+6 \mathrm{NO}_{x}(\mathrm{~g})+14.5 \mathrm{H}_{2} \mathrm{O}(\mathrm{g})\end{aligned}$ & Eq. 15 \\
\hline Ideal Weight Percent $=\frac{Z n(m I m)_{2}}{Z n O}=\frac{225 \frac{g}{m o l}}{81 \frac{g}{m o l}} x 100 \%=277 \%$ & Eq. 16 \\
\hline $\mathrm{Cu}_{3}(\mathrm{BTC})_{2}(\mathrm{~s})+16.5 \mathrm{O}_{2}(g) \rightarrow 3 \mathrm{CuO}(\mathrm{s})+18 \mathrm{CO}_{2}(g)+6 \mathrm{H}_{2} \mathrm{O}(\mathrm{g})$ & Eq. 17 \\
\hline Ideal Weight Percent $=\frac{\mathrm{Cu}_{3}(\mathrm{BTC})_{2}}{3 \mathrm{CuO}}=\frac{605 \frac{\mathrm{g}}{\mathrm{mol}}}{239 \frac{\mathrm{g}}{\mathrm{mol}}} \times 100 \%=254 \%$ & Eq. 18 \\
\hline
\end{tabular}




\section{Nitrogen Uptake Isotherms for Rapid Trials}

a)

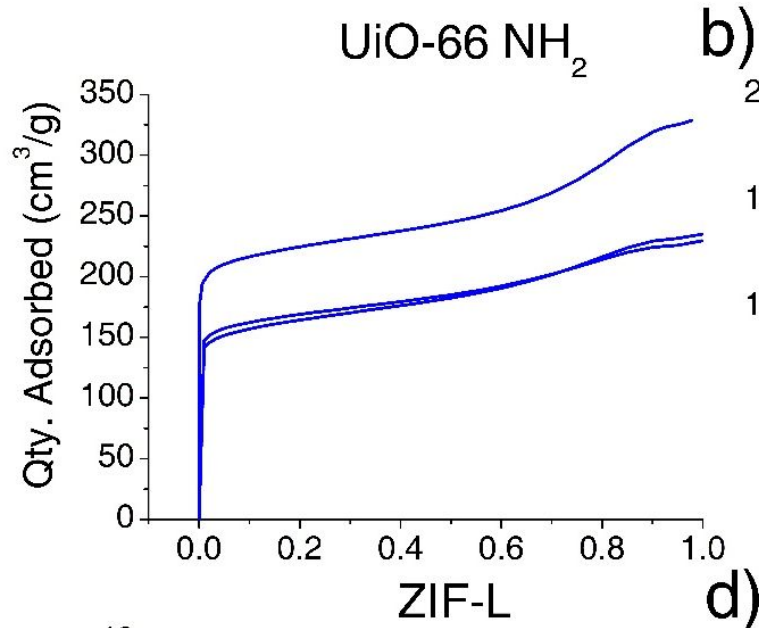

b)

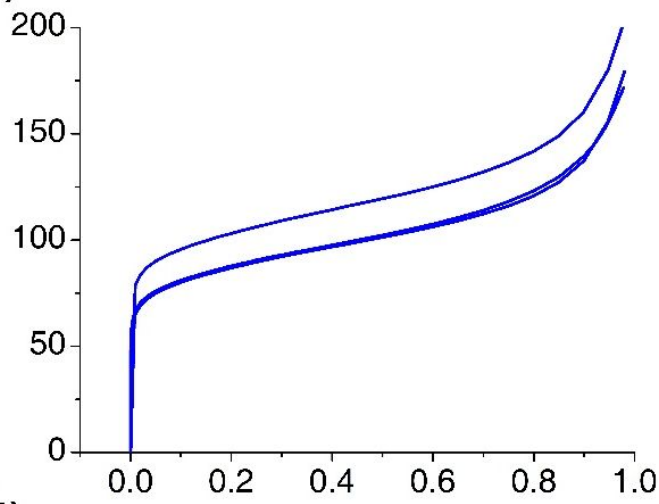

c)
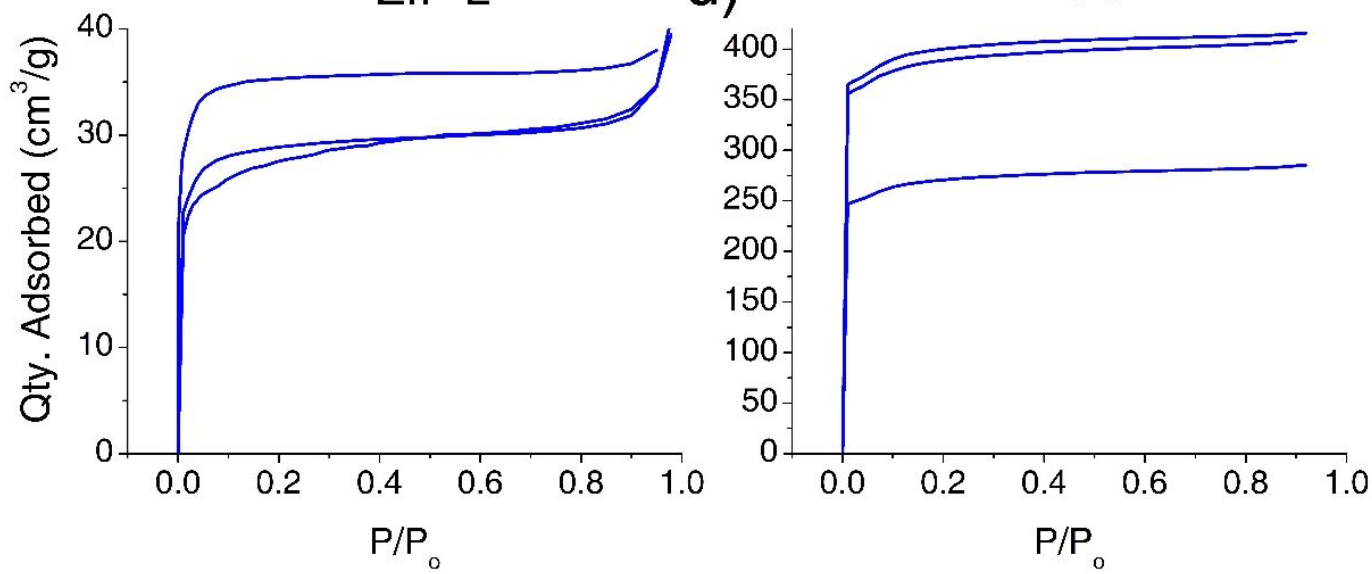

Figure S5. Nitrogen uptake isotherms of rapidly synthesized MOFs a) UiO-66 $\mathrm{NH}_{2}$ b) UiO-66 c) ZIF-L and d) HKUST-1. Isotherms were used to calculate surface area using BET theory. The isotherms for UiO-66-NH $\mathrm{H}_{2}$ and UiO-66 present some mesoporosity based on the additional uptake towards high relative pressures representing a Type II or IV isotherm. This is likely due to the presence of nanoparticle agglomerates, as observed in SEM. 


\section{Scanning Electron Microscopy Images of UiO-66- $\mathrm{NH}_{2}$ Fabric}

a)
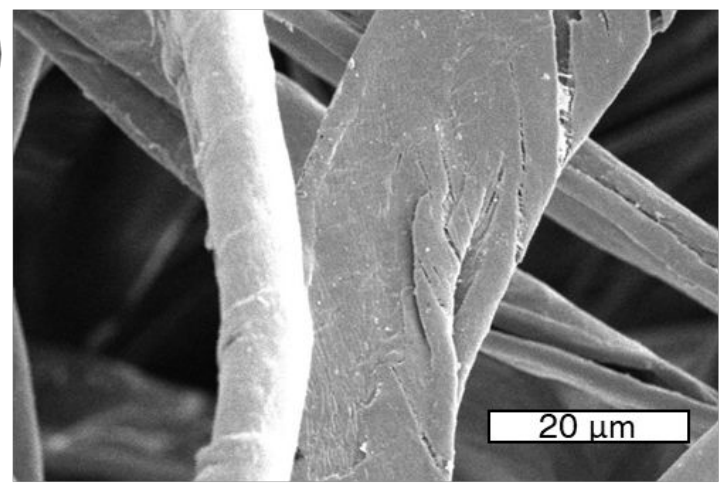

c)

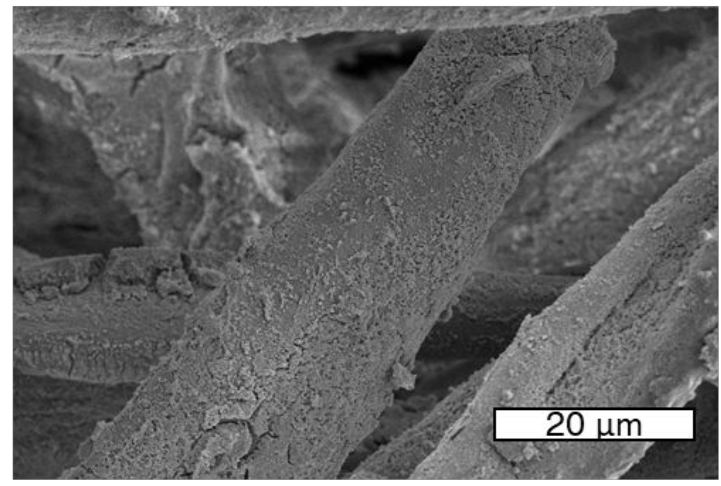

e)

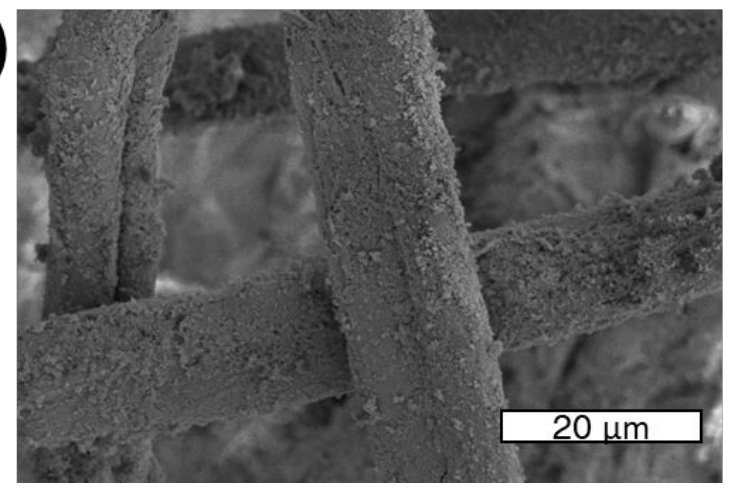

b)

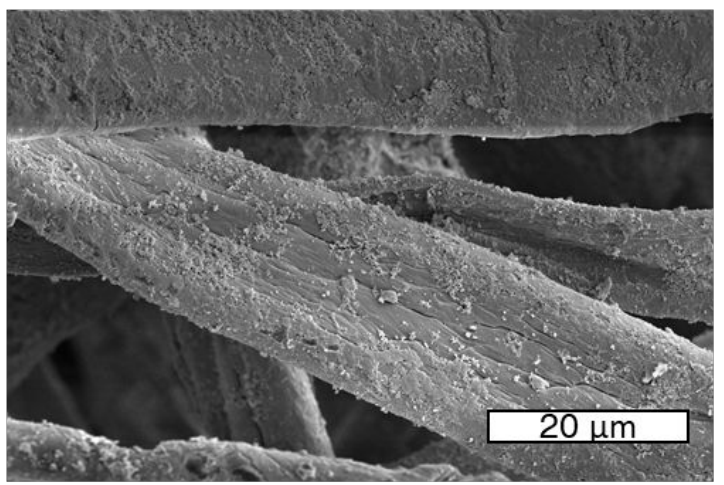

d)

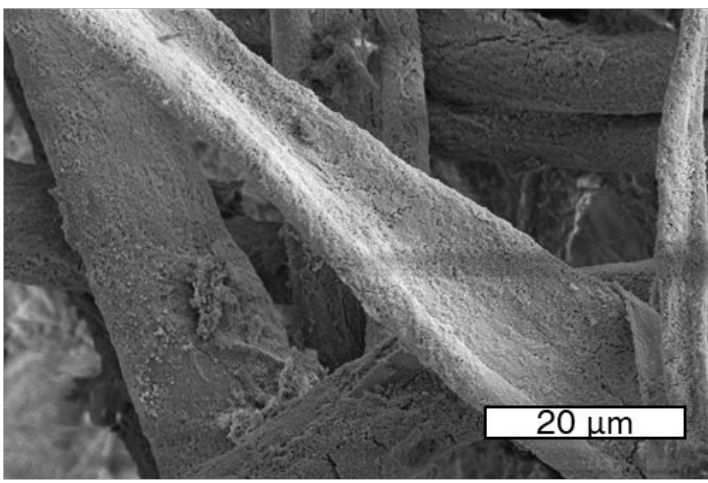

f)

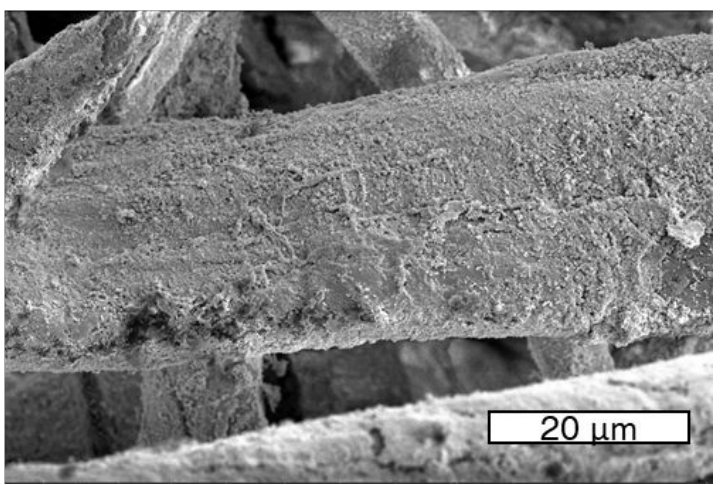

Figure S6. SEM micrographs of UiO-66- $\mathrm{NH}_{2}$ on fabric including: a) untreated cotton fabric b) 1 dip SQD UiO-66-NH $\mathrm{S}_{2}$ on cotton

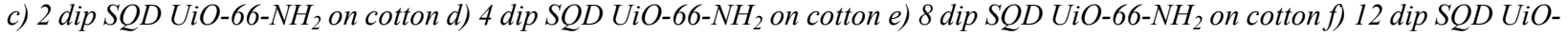
66- $\mathrm{NH}_{2}$ on cotton. Micrographs show the presence of nanoparticles on the surface of fibers, with increasing coverage as a funciton of SQD cycles. 


\section{Thermogravimetric Analysis of UiO-66 $\mathrm{NH}_{2}$ Loading on Cotton Fabric}

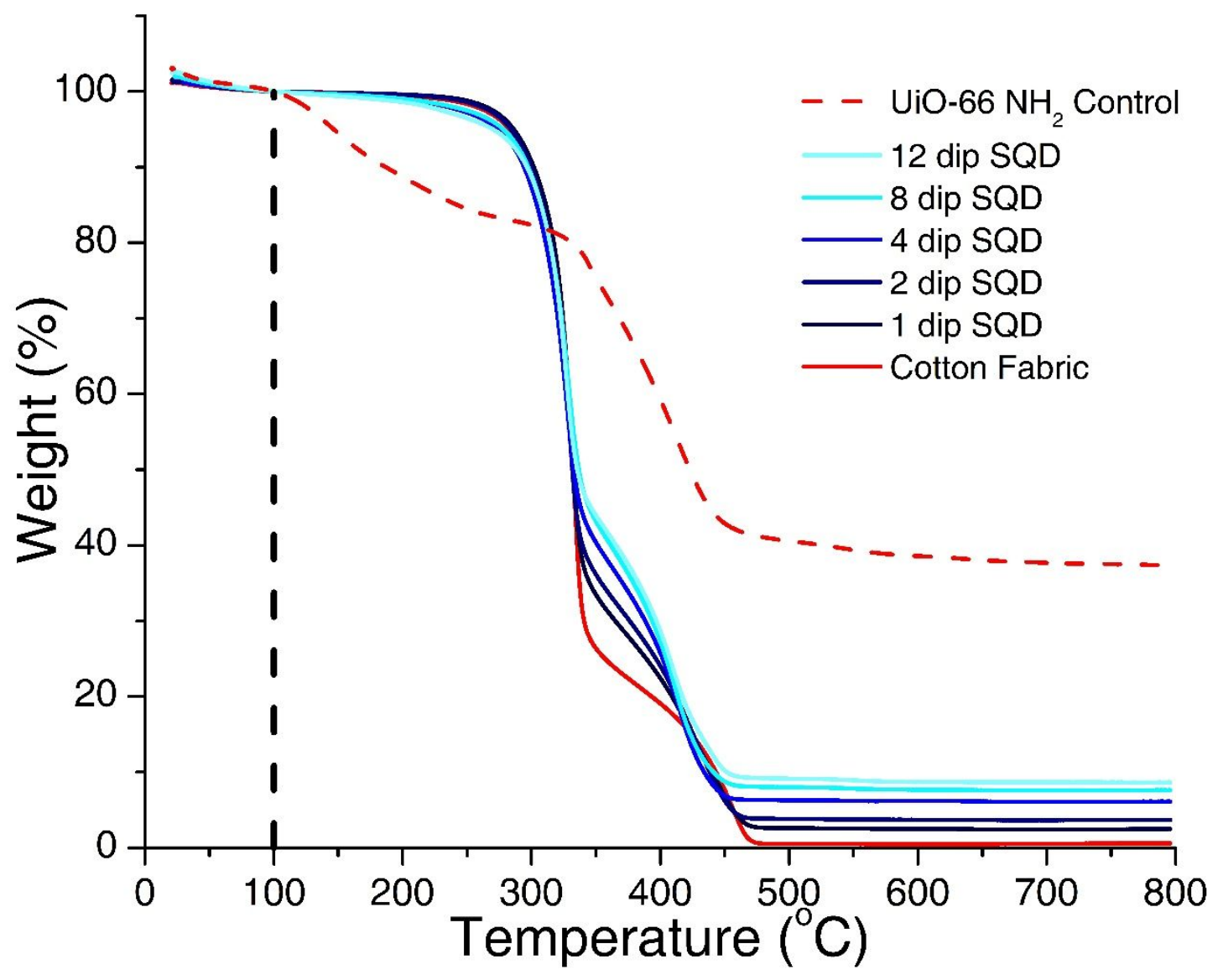

Figure S7. Thermogravimetric decomposition curves of UiO-66 $\mathrm{NH}_{2}$ (red dash), Cotton Fabric (red solid), and UiO-66-NH 2 loaded onto cotton fabric with different number of sequential dips. UiO-66- $\mathrm{NH}_{2}$ shows solvent loss up to $300{ }^{\circ} \mathrm{C}$, whereas cotton fabric and SQD show little solvent weight loss up to $300{ }^{\circ} \mathrm{C}$. The dip at $340{ }^{\circ} \mathrm{C}$ shows the decomposition of the organic cotton material, whereas the dip at $360{ }^{\circ} \mathrm{C}$ represents the decomposition of UiO-66- $\mathrm{NH}_{2}$. It can be seen as more MOF is loaded onto fabric, the decomposition curve representing UiO-66- $\mathrm{NH}_{2}$ becomes more pronounced and the remaining wt\% ( $\left.\mathrm{ZrO} \mathrm{O}_{2}\right)$ increases, indicating more metal is loaded on the fabric due to MOF loading.

A weighted average method was used to calculate the weight percent of $\mathrm{UiO}-66 \mathrm{NH}_{2}$ on cotton:

\begin{tabular}{|l|c|}
\hline$W t \% \mathrm{UiO} 6 \mathrm{NH}_{2}=\frac{Z \%-Y \%}{X \%-Y \%}$ & Eq. 19 \\
\hline
\end{tabular}

Where $\mathrm{Z} \%=$ the ash percent of Cotton@UiO-66- $\mathrm{NH}_{2}, \mathrm{Y} \%=$ Cotton fabric ash weight percent, and $\mathrm{X} \%=$ UiO-66 $\mathrm{NH}_{2}$ ash percent. 


\section{Tabulated Cotton@UiO-66- $\mathrm{NH}_{2}$ results}

Table S4. Table of wt\% ash and MOF loading as calculated from weighted average technique for different number of SQD cycles. $N=3$. Results were calculated using data shown in Supplementary Figure 7 and Supplementary Equation (19).

\begin{tabular}{|c|c|c|}
\hline SQD Dips & Wt\% ash & MOF Loading \\
\hline UiO-66 $\mathrm{NH}_{2}$ Control & $37.3 \pm 1.7 \%$ & $100 \%$ \\
\hline Cotton Control & $0.6 \%$ & $0 \%$ \\
\hline $1 \mathrm{dip}$ & $3.0 \pm 0.4 \%$ & $6.2 \pm 1.3 \%$ \\
\hline $2 \mathrm{dip}$ & $3.9 \pm 0.2 \%$ & $8.9 \pm 1.0 \%$ \\
\hline $4 \mathrm{dip}$ & $5.0 \pm 0.9 \%$ & $12.1 \pm 3.1 \%$ \\
\hline $8 \mathrm{dip}$ & $7.3 \pm 0.3 \%$ & $18.2 \pm 1.8 \%$ \\
\hline $12 \mathrm{dip}$ & $8.2 \pm 0.8 \%$ & $20.7 \pm 3.2 \%$ \\
\hline
\end{tabular}

\section{MOF Conversion Calculations}

All syntheses were performed using a metal to linker ratio matching stoichiometry in a pristine MOF, where the pristine formula for each MOF is shown below:

Table S5. Table showing the pristine formulas of prototypical MOFs used to calculate conversion

\begin{tabular}{|c|c|}
\hline MOF Name & Pristine Chemical Formula \\
\hline UiO-66- $\mathrm{NH}_{2}$ & $\mathrm{Zr}_{6} \mathrm{O}_{4}(\mathrm{OH})_{4}(\mathrm{ATA})_{6}$ \\
\hline $\mathrm{UiO}-66$ & $\mathrm{Zr}_{6} \mathrm{O}_{4}(\mathrm{OH})_{4}(\mathrm{BDC})_{6}$ \\
\hline $\mathrm{ZIF}-\mathrm{L}$ & $\mathrm{Zn}(\mathrm{mim})_{2} \cdot(\mathrm{Hmim})_{1 / 2} \cdot 1.5 \mathrm{H}_{2} \mathrm{O}$ \\
\hline $\mathrm{HKUST}-1$ & $\mathrm{Cu}_{3}(\mathrm{BTC})_{2} \cdot 3 \mathrm{H}_{2} \mathrm{O}$ \\
\hline
\end{tabular}

Assuming all deprotonated linker and metal node component can participate in a reaction, $100 \%$ conversion is calculated via mass balance of metal (or metal oxide) nodes and deprotonated linkers. An example calculation for UiO-66 $\mathrm{NH}_{2}$ is shown below:

$9.1242 \mathrm{~g}$ Metal Solution $\times 0.069 \frac{\mathrm{g} \mathrm{ZrOCl}_{2} \cdot 8 \mathrm{H}_{2} \mathrm{O}}{\mathrm{g} \mathrm{Metal} \mathrm{Solution}} \times \frac{1 \mathrm{~mol} \mathrm{ZrOCl}_{2} \cdot 8 \mathrm{H}_{2} \mathrm{O}}{322.25 \mathrm{~g} \mathrm{ZrOCl}_{2} \cdot 8 \mathrm{H}_{2} \mathrm{O}}$

$$
x \frac{1 \mathrm{~mol} \mathrm{Zr}_{6} \mathrm{O}_{4}(\mathrm{OH})_{4}}{6 \mathrm{~mol} \mathrm{ZrOCl}_{2} \cdot 8 \mathrm{H}_{2} \mathrm{O}} x \frac{679.34 \mathrm{~g} \mathrm{Zr}_{6} \mathrm{O}_{4}(\mathrm{OH})_{4}}{1 \mathrm{~mol} \mathrm{Zr}_{6} \mathrm{O}_{4}(\mathrm{OH})_{4}}=0.2212 \mathrm{~g} \mathrm{Zr}_{6} \mathrm{O}_{4}(\mathrm{OH})_{4}
$$

Eq. 20

$10.3171 \mathrm{~g}$ Linker Solution $\times 0.034 \frac{\mathrm{g} \mathrm{H}_{2} A T A}{\text { g Linker Solution }} \times \frac{179.15 \mathrm{~g} \mathrm{ATA} A^{2-}}{181.15 \mathrm{~g} \mathrm{H}_{2} A T A}$

Eq. 21

$$
=0.3510 \mathrm{~g} A T A^{2-}
$$


Therefore, $100 \%$ conversion of all reactants:

$$
0.2212 \mathrm{~g} \mathrm{Zr}_{6} \mathrm{O}_{4}(\mathrm{OH})_{4}+0.3510 \mathrm{~g} \mathrm{ATA}^{2-}=0.5722 \mathrm{~g} \mathrm{Zr}_{6} \mathrm{O}_{6}(\mathrm{ATA})_{x} \quad \text { Eq. } 22
$$

Where $x$ depends on the number of linker defects present in the precipitate. It should be noted the HmIm was the limiting reagent for the ZIF-L reaction, therefore conversion was calculated with respect to HmIm. 


\section{Tabulated Yield Results}

Table S6. Table of $\mathrm{pH}$ value after 10-minute reaction time, \% conversion, \% crystallinity, and \% yield for rapidly synthesized MOFs and controls. $N=3$ syntheses. Crystallinity is assumed to be $100 \%$ if conversion provided insufficient material for PXRD, representing an upper bound for MOF obtained.

\begin{tabular}{|c|c|c|c|c|}
\hline MOF & pH@10 Minutes & \% Conversion & \% Crystallinity & \% Yield \\
\hline \multirow{11}{*}{ UiO-66-NH } & $2.5 \pm 0.1$ & $4 \pm 1$ & N/A (1) & $4 \pm 1$ \\
\hline & $2.7 \pm 0.1$ & $18 \pm 10$ & $\mathrm{~N} / \mathrm{A}(1)$ & $18 \pm 10$ \\
\hline & $3.4 \pm 0.1$ & $96 \pm 1$ & $42 \pm 4$ & $40 \pm 4$ \\
\hline & $3.9 \pm 0.1$ & $96 \pm 1$ & $38 \pm 3$ & $37 \pm 3$ \\
\hline & $4.0 \pm 0.1$ & $96 \pm 1$ & $40 \pm 1$ & $39 \pm 1$ \\
\hline & $4.4 \pm 0.1$ & $92 \pm 2$ & $37 \pm 2$ & $34 \pm 3$ \\
\hline & $4.7 \pm 0.1$ & $84 \pm 1$ & $30 \pm 4$ & $26 \pm 3$ \\
\hline & $5.1 \pm 0.1$ & $81 \pm 7$ & $18 \pm 1$ & $14 \pm 2$ \\
\hline & $6.2 \pm 0.4$ & $62 \pm 3$ & 0 & 0 \\
\hline & $13.2 \pm 0.4$ & $47 \pm 1$ & 0 & 0 \\
\hline & Control & $38 \pm 4$ & $47 \pm 2$ & $18 \pm 3$ \\
\hline \multirow[t]{9}{*}{ UiO-66 } & $2.9 \pm 0.1$ & $4 \pm 1$ & N/A (1) & $4 \pm 1$ \\
\hline & $3.7 \pm 0.1$ & $9 \pm 1$ & N/A (1) & $9 \pm 1$ \\
\hline & $4.3 \pm 0.1$ & $34 \pm 4$ & $22 \pm 2$ & $8 \pm 2$ \\
\hline & $4.9 \pm 0.1$ & $79 \pm 2$ & $17 \pm 4$ & $13 \pm 3$ \\
\hline & $5.1 \pm 0.1$ & $74 \pm 2$ & $13 \pm 2$ & $10 \pm 2$ \\
\hline & $5.2 \pm 0.1$ & $71 \pm 3$ & $14 \pm 5$ & $10 \pm 4$ \\
\hline & $6.8 \pm 0.3$ & $60 \pm 2$ & 0 & 0 \\
\hline & $12.5 \pm 0.3$ & $40 \pm 1$ & 0 & 0 \\
\hline & Control & $104 \pm 5$ & $44 \pm 1$ & $46 \pm 3$ \\
\hline \multirow[t]{8}{*}{ ZIF-L } & $7.0 \pm 0.1$ & $13 \pm 1$ & N/A (1) & $13 \pm 1$ \\
\hline & $7.4 \pm 0.1$ & $89 \pm 8$ & $49 \pm 2$ & $44 \pm 5$ \\
\hline & $7.6 \pm 0.1$ & $102 \pm 3$ & $48 \pm 1$ & $49 \pm 3$ \\
\hline & $7.8 \pm 0.3$ & $103 \pm 7$ & $46 \pm 2$ & $48 \pm 5$ \\
\hline & $8.3 \pm 0.3$ & $107 \pm 1$ & $49 \pm 1$ & $53 \pm 2$ \\
\hline & $8.2 \pm 0.2$ & $108 \pm 2$ & $43 \pm 8$ & $47 \pm 9$ \\
\hline & $9.1 \pm 1.0$ & $107 \pm 1$ & $21 \pm 18$ & $22 \pm 19$ \\
\hline & Control & $71 \pm 1$ & $59 \pm 1$ & $42 \pm 1$ \\
\hline \multirow[t]{10}{*}{ HKUST-1 } & $2.0 \pm 0.1$ & $49 \pm 11$ & $45 \pm 4$ & $22 \pm 7$ \\
\hline & $3.3 \pm 0.1$ & $86 \pm 4$ & $58 \pm 1$ & $50 \pm 3$ \\
\hline & $3.8 \pm 0.1$ & $99 \pm 2$ & $42 \pm 1$ & $41 \pm 1$ \\
\hline & $4.1 \pm 0.1$ & $100 \pm 2$ & $34 \pm 2$ & $34 \pm 2$ \\
\hline & $4.9 \pm 0.1$ & $100 \pm 1$ & $22 \pm 1$ & $23 \pm 2$ \\
\hline & $5.2 \pm 0.1$ & $97 \pm 1$ & $19 \pm 1$ & $18 \pm 1$ \\
\hline & $5.7 \pm 0.1$ & $95 \pm 2$ & $12 \pm 4$ & $12 \pm 4$ \\
\hline & $6.5 \pm 0.2$ & $89 \pm 1$ & 0 & 0 \\
\hline & $7.0 \pm 0.1$ & $75 \pm 5$ & 0 & 0 \\
\hline & Control & $83 \pm 3$ & $84 \pm 1$ & $69 \pm 3$ \\
\hline
\end{tabular}




\section{Pollution Capture Data}

Table S7. Table of downstream pollution concentration data used to calculate capture efficiency and capture efficiency of control cotton filter and varied $S Q D$ cycles. $N=3$.

\begin{tabular}{|c|c|c|}
\hline Sample & $\begin{array}{c}\text { Average Downstream Concentration } \\
\left(\boldsymbol{\mu g} / \mathbf{m}^{\mathbf{3}}\right)\end{array}$ & \% Capture \\
\hline No Filter & $506 \pm 35$ & 0 \\
\hline Cotton Control & $240 \pm 14$ & $53 \pm 7$ \\
\hline 1 dip & $178 \pm 17$ & $65 \pm 11$ \\
\hline $2 \operatorname{dip}$ & $159 \pm 1$ & $69 \pm 5$ \\
\hline $4 \operatorname{dip}$ & $111 \pm 7$ & $78 \pm 10$ \\
\hline 8 dip & $81 \pm 4$ & $84 \pm 10$ \\
\hline $12 \operatorname{dip}$ & $78 \pm 5$ & $85 \pm 11$ \\
\hline
\end{tabular}

\section{Supplementary Information References}

(1) Pakamorè, I.; Rousseau, J.; Rousseau, C.; Monflier, E.; Szilágyi, P. Á. An Ambient-Temperature Aqueous Synthesis of Zirconium-Based Metal-Organic Frameworks. Green Chem. 2018, 20 (23), 5292-5298. https://doi.org/10.1039/C8GC02312C.

(2) Cavka, J. H.; Jakobsen, S.; Olsbye, U.; Guillou, N.; Lamberti, C.; Bordiga, S.; Lillerud, K. P. A New Zirconium Inorganic Building Brick Forming Metal Organic Frameworks with Exceptional Stability. J. Am. Chem. Soc. 2008, 130 (42), 13850-13851. https://doi.org/10.1021/ja8057953.

(3) Katz, M. J.; Brown, Z. J.; Colón, Y. J.; Siu, P. W.; Scheidt, K. A.; Snurr, R. Q.; Hupp, J. T.; Farha, O. K. A Facile Synthesis of UiO-66, UiO-67 and Their Derivatives. Chem. Commun. 2013, 49 (82), 9449. https://doi.org/10.1039/c3cc46105j.

(4) Kim, H. K.; Yun, W. S.; Kim, M.-B.; Kim, J. Y.; Bae, Y.-S.; Lee, J.; Jeong, N. C. A Chemical Route to Activation of Open Metal Sites in the Copper-Based Metal-Organic Framework Materials HKUST-1 and Cu-MOF-2. J. Am. Chem. Soc. 2015, 137 (31), 10009-10015. https://doi.org/10.1021/jacs.5b06637.

(5) Chen, R.; Yao, J.; Gu, Q.; Smeets, S.; Baerlocher, C.; Gu, H.; Zhu, D.; Morris, W.; Yaghi, O. M.; Wang, H. A Two-Dimensional Zeolitic Imidazolate Framework with a Cushion-Shaped Cavity for CO2 Adsorption. Chem. Commun. 2013, 49 (82), 9500. https://doi.org/10.1039/c3cc44342f.

(6) Degen, T.; Sadki, M.; Bron, E.; König, U.; Nénert, G. The HighScore Suite. Powder Diffr. 2014, 29 (S2), S13-S18. https://doi.org/10.1017/S0885715614000840.

(7) Reinsch, H.; Waitschat, S.; Chavan, S. M.; Lillerud, K. P.; Stock, N. A Facile "Green" Route for Scalable Batch Production and Continuous Synthesis of Zirconium MOFs: A Facile "Green" Route for Scalable Batch Production and Continuous Synthesis of Zirconium MOFs. Eur. J. Inorg. Chem. 2016, 2016 (27), 4490-4498. https://doi.org/10.1002/ejic.201600295.

(8) Gökpinar, S.; Diment, T.; Janiak, C. Environmentally Benign Dry-Gel Conversions of Zr-Based UiO Metal-Organic Frameworks with High Yield and the Possibility of Solvent Re-Use. Dalton Trans. 2017, 46 (30), 9895-9900. https://doi.org/10.1039/C7DT01717K.

(9) Avci-Camur, C.; Troyano, J.; Pérez-Carvajal, J.; Legrand, A.; Farrusseng, D.; Imaz, I.; Maspoch, D. Aqueous Production of Spherical Zr-MOF Beads via Continuous-Flow Spray-Drying. Green Chem. 2018, 20 (4), 873-878. https://doi.org/10.1039/C7GC03132G.

(10) Rubio-Martinez, M.; Batten, M. P.; Polyzos, A.; Carey, K.-C.; Mardel, J. I.; Lim, K.-S.; Hill, M. R. Versatile, High Quality and Scalable Continuous Flow Production of Metal-Organic Frameworks. Sci. Rep. 2015, 4 (1), 5443. https://doi.org/10.1038/srep05443. 
(11) Li, Y.; Liu, Y.; Gao, W.; Zhang, L.; Liu, W.; Lu, J.; Wang, Z.; Deng, Y.-J. Microwave-Assisted Synthesis of UIO-66 and Its Adsorption Performance towards Dyes. CrystEngComm 2014, 16 (30), 7037-7042. https://doi.org/10.1039/C4CE00526K.

(12) Carné-Sánchez, A.; Imaz, I.; Cano-Sarabia, M.; Maspoch, D. A Spray-Drying Strategy for Synthesis of Nanoscale Metal-Organic Frameworks and Their Assembly into Hollow Superstructures. Nat. Chem. 2013, 5 (3), 203-211. https://doi.org/10.1038/nchem.1569.

(13) Zhao, J.; Nunn, W. T.; Lemaire, P. C.; Lin, Y.; Dickey, M. D.; Oldham, C. J.; Walls, H. J.; Peterson, G. W.; Losego, M. D.; Parsons, G. N. Facile Conversion of Hydroxy Double Salts to Metal-Organic Frameworks Using Metal Oxide Particles and Atomic Layer Deposition Thin-Film Templates. J. Am. Chem. Soc. 2015, 137 (43), 13756-13759. https://doi.org/10.1021/jacs.5b08752.

(14) Gimeno-Fabra, M.; Munn, A. S.; Stevens, L. A.; Drage, T. C.; Grant, D. M.; Kashtiban, R. J.; Sloan, J.; Lester, E.; Walton, R. I. Instant MOFs: Continuous Synthesis of Metal-Organic Frameworks by Rapid Solvent Mixing. Chem. Commun. 2012, 48 (86), 10642. https://doi.org/10.1039/c2cc34493a.

(15) Huo, J.; Brightwell, M.; El Hankari, S.; Garai, A.; Bradshaw, D. A Versatile, Industrially Relevant, Aqueous Room Temperature Synthesis of HKUST-1 with High Space-Time Yield. $J$. Mater. Chem. A 2013, 1 (48), 15220. https://doi.org/10.1039/c3ta14409g.

(16) Garcia Marquez, A.; Horcajada, P.; Grosso, D.; Ferey, G.; Serre, C.; Sanchez, C.; Boissiere, C. Green Scalable Aerosol Synthesis of Porous Metal-Organic Frameworks. Chem. Commun. 2013, 49 (37), 3848. https://doi.org/10.1039/c3cc39191d.

(17) Czaja, A. U.; Trukhan, N.; Müller, U. Industrial Applications of Metal-Organic Frameworks. Chem. Soc. Rev. 2009, 38 (5), 1284. https://doi.org/10.1039/b804680h.

(18) Faustini, M.; Kim, J.; Jeong, G.-Y.; Kim, J. Y.; Moon, H. R.; Ahn, W.-S.; Kim, D.-P. Microfluidic Approach toward Continuous and Ultrafast Synthesis of Metal-Organic Framework Crystals and Hetero Structures in Confined Microdroplets. J. Am. Chem. Soc. 2013, 135 (39), 14619-14626. https://doi.org/10.1021/ja4039642.

(19) Pan, Y.; Liu, Y.; Zeng, G.; Zhao, L.; Lai, Z. Rapid Synthesis of Zeolitic Imidazolate Framework-8 (ZIF-8) Nanocrystals in an Aqueous System. Chem. Commun. 2011, 47 (7), 2071-2073.

https://doi.org/10.1039/C0CC05002D. 